\title{
Формирование и исследование локально-растянутых Ge-микроструктур для кремниевой фотоники
}

\author{
(C) А.В. Новиков ${ }^{1,2}$, Д.В. Юрасов ${ }^{1}$, Е.Е. Морозова ${ }^{1}$, Е.В. Скороходов ${ }^{1}$, В.А. Вербус ${ }^{1,3}$, \\ А.Н. Яблонский ${ }^{1}$, Н.А. Байдакова ${ }^{1}$, Н.С. Гусев ${ }^{1}$, К.Е. Кудрявцев ${ }^{1,2}$, \\ A.B. Нежданов ${ }^{2}$, А.И. Машин ${ }^{2}$ \\ ${ }^{1}$ Институт ффизики микроструктур Российской академии наук, \\ 603087 д. Афронино, Нижегородская обл., Россия \\ ${ }^{2}$ Нижегородский государственный университет им. Н.И. Лобачевского, \\ 603950 Нижний Новгород, Россия \\ ${ }^{3}$ НИУ Высшая школа экономики, \\ 603155 Нижний Новгород, Россия \\ E-mail: anov@ipmras.ru
}

(Получена 25 апреля 2018 г. Принята к печати 7 мая 2018 г.)

\begin{abstract}
Представлены результаты по формированию и исследованию локально-растянутых Ge-микроструктур (,микромостиков“) на основе слоев Ge, выращенных на кремниевых подложках. Выполнен теоретический анализ распределения деформации в свободновисящих Ge-микромостиках, который выявил, что для достижения максимального уровня деформации растяжения в самом микромостике необходима минимизация концентрации упругих напряжений во всех углах формируемой микроструктуры. Измерения локальной деформации методом комбинационного рассеяния света показали увеличение деформации растяжения с $0.2-0.25 \%$ в исходной Ge-пленке до $2.4 \%$ в Ge-микромостиках. Методом спектроскопии микрофотолюминесценции продемонстрировано значительное возрастание интенсивности и существенная модификация спектра ФЛ в области максимальных растягивающих напряжений в Ge-микромостиках и их окрестностях по сравнению со слабо растянутыми участками исходных слоев $\mathrm{Ge}$.
\end{abstract}

DOI: 10.21883/FTP.2018.11.46594.16

\section{1. Введение}

Германий благодаря небольшой ( 140 мэВ) разнице энергий между Г- и $L$-долинами в зоне проводимости, a также совместимости с современной $\mathrm{Si}$ интегральной технологией в последние несколько лет привлекает к себе повышенное внимание как перспективный материал для создания источников излучения ближнего ИК диапазона для кремниевой оптоэлектроники $[1,2]$. Для повышения эффективности излучательной рекомбинации носителей заряда в Ge необходимо уменьшать энергетический зазор между его прямой и непрямой запрещенными зонами. Легирование Ge-донорами до уровней, существенно превышающих $10^{19} \mathrm{~cm}^{-3}$, за счет заполнения электронных состояний в $L$-долине позволяет частично решить эту задачу [3,4]. Но кардинальное уменьшение разницы между энергиями прямой и непрямой запрещенных зон может быть достигнуто за счет приложения к Ge растягивающих напряжений [3]. Однако, согласно расчетам, величина деформации, при которой разница энергий между $\Gamma$ - и $L$-долинами уменьшается до нуля и $\mathrm{Ge}$ становится прямозонным полупроводником, достаточно велика и составляет $1.5-2 \%$ для двухосной и 4.5-5\% для одноосной деформации [3-5].

Указанные величины деформации при формировании Ge на кремниевых подложках могут быть реализованы при использовании промежуточных $\mathrm{GeSnSi}$ релаксированных слоев, имеющих параметр кристаллической решетки больше, чем у $\mathrm{Ge}[4,6]$. Однако при таком подходе возникают существенные проблемы, связанные с формированием GeSnSi-релаксированных слоев высокого кристаллического качества. В то же время при росте Ge непосредственно на $\mathrm{Si}(001)$-подложках за счет разницы коэффициентов температурного расширения $\mathrm{Ge}$ и $\mathrm{Si}$ возможно получение растянутых слоев $\mathrm{Ge}$ с максимальной деформацией до $\sim 0.3 \%$ [7]. Данные малые величины однородного, двухосного растяжения за счет так называемого метода „концентрации напряжений“ могут быть использованы для создания локальнорастянутых Ge-микроструктур с высокими значениями деформации $[8,9]$. Суть метода „концентрации напряжений состоит в перераспределении однородных упругих напряжений в свободновисящих пленках, в которых происходит релаксация упругих напряжений в большей части микроструктуры и многократное увеличение деформации в специально созданных малых областях, например в микромостиках [8,9]. В недавней работе [10] было доложено о наблюдении лазерной генерации на локально-растянутом данным методом Ge-микромостике. Генерация была достигнута при низких температурах $(\sim 83 \mathrm{~K})$ при оптической накачке мостика. Однако при комнатной температуре до сих пор лазерная генерация в Ge-микроструктурах не наблюдалась, что указывает на недостаточную изученность влияния различных параметров локально деформированных $\mathrm{Ge} / \mathrm{Si}(001)$-микромостиков на их структурные и оптические свойства и подтверждает актуальность проведения дальнейших исследований в данной области. 
В настоящей работе приведены результаты по особенностям формирования свободновисящих Ge-микромостиков на подложках $\mathrm{Si}(001)$ и „кремний-на-изоляторе“, экспериментальному и теоретическому анализу распределения деформации в полученных структурах и исследованию их излучательных свойств.

\section{2. Методика эксперимента}

Исходными структурами для формирования напряженных (растянутых) микромостиков служили релаксированные слои $\mathrm{Ge}$, выращенные методом молекулярнопучковой эпитаксии на подложках $\mathrm{Si}(001)$ и „кремнийна-окисле“ („silicon-on-insulator“, SOI). Для получения $\mathrm{Ge}-$ слоев с низкой концентрацией прорастающих дефектов и малой шероховатостью поверхности использовался двухтемпературный режим роста и циклический отжиг выращенных структур [11-13]. За счет оптимизации условий роста и параметров отжига плотность прорастающих дислокаций в полученных Ge-буферах толщиной $\sim 500$ нм находилась на уровне $(2-5) \cdot 10^{7} \mathrm{~cm}^{-2}$, а шероховатость поверхности была меньше 1 нм [14]. Как было отмечено во Введении, легирование Ge-донорными примесями повышает вероятность прямых излучательных переходов в нем. Поэтому часть Ge-структур, используемых для формирования микромостиков, помимо нелегированного Ge-буфера толщиной $\sim 500$ нм имела на поверхности слой $\mathrm{Ge}$, легированный донорной

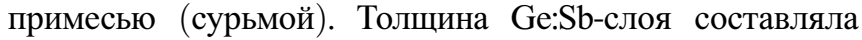
$300-400$ нм. При формировании легированных Ge-слоев использовался ранее развитый авторами метод легирования Ge-сегрегирующими примесями [15]. Концентрация электронов в легированных слоях, по данным измерений эффекта Холла, лежала в диапазоне $(2-5) \cdot 10^{19} \mathrm{~cm}^{-3}$ и совпадала с концентрацией введенной сурьмы, определенной методом вторичной ионной масс-спектрометрии, что говорит о полной электрической активации примеси. По данным рентгенодифракционного анализа, выращенные слои Ge характеризовались двумерной деформацией растяжения на уровне $0.2-0.25 \%$, вызванной разницей в коэффициентах термического расширения $\mathrm{Si}$ и $\mathrm{Ge}$ [7].

Создание свободновисящих $\mathrm{Ge}$-микромостиков (рис. 1) осуществлялось методами оптической литографии, плазмохимического и селективного химического травления. На первом этапе с помощью безмасочной, лазерной оптической литографии на установке Heidelberg uPG 101 в фоторезисте AZ1512 формировался необходимый рисунок для плазмохимического травления. Применение безмасочной литографии позволяло оперативно менять дизайн создаваемых микроструктур. На втором этапе в смеси газов $\mathrm{SF}_{6} / \mathrm{C}_{4} \mathrm{~F}_{8}$ с использованием фоторезиста в качестве маски выполнялось плазмохимическое травление (ПХТ) структуры. Использование смеси газов $\mathrm{SF}_{6} / \mathrm{C}_{4} \mathrm{~F}_{8}$ и подбор условий травления обеспечивали высокую анизотропию травления в вертикальном направлении. Для структур, выращенных на

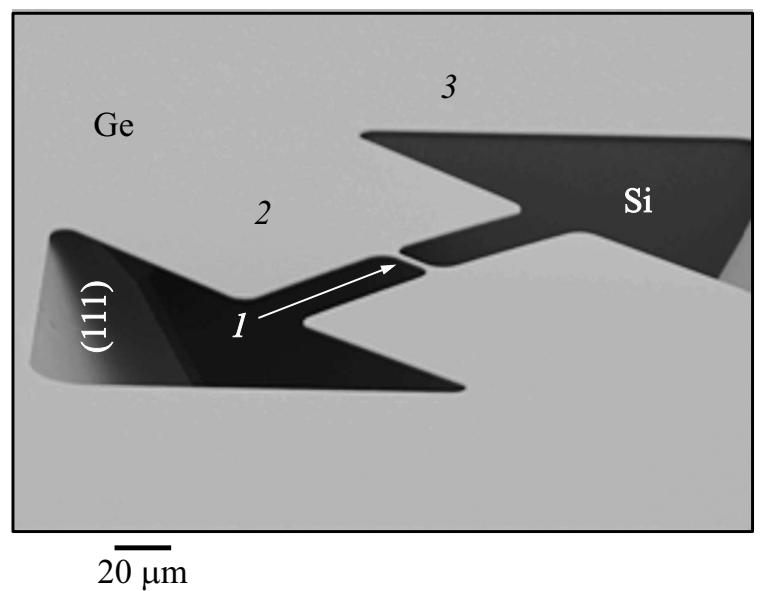

Рис. 1. СЭМ-снимок сформированного Ge-микромостика. Цифрами обозначены: 1 - микромостик, 2 - подвешенная часть Ge-слоя („берег“), 3 - исходный слой $\mathrm{Ge}$, не подвергавшийся обработке. Также на рисунке обозначена одна из плоскостей семейства (111), ограничивающая подвешенную часть микроструктуры.

$\mathrm{Si}(001)$-подложках, травление проходило на глубину, которая на 200-300 нм превышала толщину Ge-слоя. Для структур, сформированных на подложках SOI, травление проводилось до скрытого слоя окисла. Таким образом, в результате ПХТ формировался нужный дизайн микроструктуры и осуществлялся доступ к слоям $\mathrm{Si}$ и $\mathrm{SiO}_{2}$, находящимся под $\mathrm{Ge}$, для проведения последующего их селективного травления. На финальном этапе осуществлялось „Подвешивание“ Ge-микромостиков за счет селективного травления либо $\mathrm{Si}$ в структурах, выращенных на $\mathrm{Si}(001)$, либо $\mathrm{Si}$ и $\mathrm{SiO}_{2}$ в структурах, сформированных на SOI подложках. Селективное травление кремния проводилось в 20\% водном растворе КОН при температуре $55-65^{\circ} \mathrm{C}$. Водный раствор КОН обладает высокой селективностью травления по отношению к SiGe-сплаву с долей $\mathrm{Ge}$ более $10 \%$ [16]. Отличительной особенностью травления кремния в КОН является высокая анизотропия травления, которая может быть использована при формировании Ge-микромостиков с фиксированной величиной деформации. Травление окисла проводилось в растворе $\mathrm{NH}_{4} \mathrm{~F}: \mathrm{HF}: \mathrm{H}_{2} \mathrm{O}$ (Buffered Oxide Etch, BOE), который имеет высокую селективность как по отношению к кремнию, так и по отношению к SiGe-сплавам. Травление окисла в ВОЕ является изотропным.

Полученные Ge-микроструктуры анализировались c помощью сканирующей электронной микроскопии $(\mathrm{CЭM).} \mathrm{Измерения} \mathrm{локальной} \mathrm{деформации} \mathrm{были} \mathrm{вы-}$ полнены методом спектроскопии комбинационного рассеяния света с высоким ( 1 мкм) пространственным разрешением (метод микро-КРС). Измерения методом микро-КРС проводились с помощью зондовой нанолаборатории NTEGRA Spectra (3AO „НT-МДТ“). При измерениях микро-КРС особое внимание уделялось минимизации мощности используемого лазерного возбуж- 
дения для предотвращения разогрева свободновисящих микроструктур. Величина и знак деформации определялись по смещению пика LO-фонона Ge в структурах относительно положения этого пика в объемном, недеформированном Ge [17].

Расчеты распределения деформации в Ge-микроструктурах были выполнены методом конечных элементов (FEM). Были рассчитаны трехмерное распределение всех компонент тензора деформации, а также распределения главных деформаций в структуре. В расчетах был использован тензор модулей упругости германия со значениями $c_{11}=129.2$ ГПа, $c_{12}=47.9$ ГПа и $c_{44}=67.0$ ГПа [18]. В качестве исходной, однородной деформации растяжения использовалось значение $\varepsilon=0.25 \%$, определенное из рентгенодифракционного

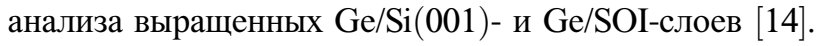

Для исследования излучательных свойств полученных локально-растянутых Ge-микроструктур применялась методика спектроскопии микрофотолюминесценции (микро-ФЛ). В данной методике сигнал ФЛ возбуждался излучением второй гармоники Nd:YAG-лазера $(532$ нм). Лазерный луч с помощью объектива $\times 50$ фокусировался на образце в пятно диаметром $\sim 2$ мкм. Для сбора сигнала ФЛ исследуемых структур использовался тот же объектив. Подведение возбуждающего лазерного луча и вывод сигнала ФЛ исследуемых структур осуществлялись по нормали к поверхности образца. Сигнал ФЛ регистрировался с использованием решеточного монохроматора Acton 2300i и многоканального детектора OMA-V на основе линейки фотодиодов InGaAs (0.8-2.2 мкм). Все люминесцентные измерения были выполнены при комнатной температуре.

\section{3. Результаты и их обсуждение}

В работе теоретически и экспериментально исследовались Ge-микромостики различного дизайна и с различными параметрами. СЭМ снимок одного из типов анализируемых и изготавливаемых Ge-микромостиков представлен на рис. 1. На рис. 2 приведены модели подобного мостика, используемые в расчетах распределения деформации при подвешивании микромостика за счет анизотропного травления кремния (рис. 2, $a$ ) или изотропного травления $\mathrm{SiO}_{2}$ (рис. 2,b). Общей чертой всех мостиков была их ориентация вдоль кристаллографического направления типа [100] (рис. 1). Именно одноосное растяжение $\mathrm{Ge}$ в этом направлении позволяет достичь наибольшего уменьшения энергетической разницы между прямой и непрямой запрещенными зонами $\mathrm{Ge}$ при фиксированной величине деформации $[19,20]$. Основными параметрами, которые определяют величину деформации микромостика, являются исходная деформация $\mathrm{Ge}-$ слоя $\left(\varepsilon_{0}\right)$, ширина $(w)$ и длина $(l)$ мостиков, общая длина подвешенной микроструктуры $(L)$ и ширина подвешенных частей Ge-пленки („берегов“") $(W)$ (рис. 1 и 2) [9]. Деформация в мостике растет с

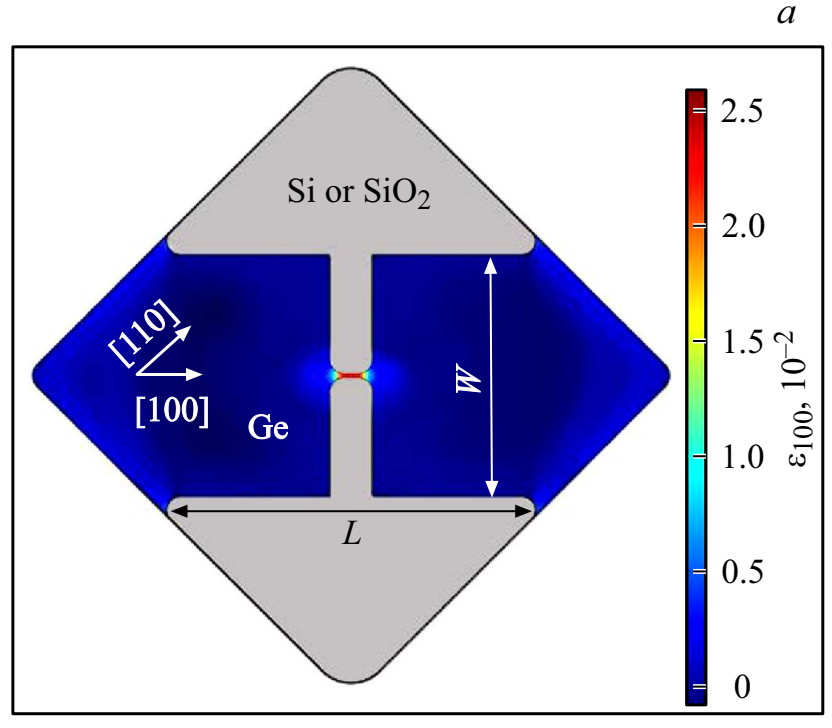

b

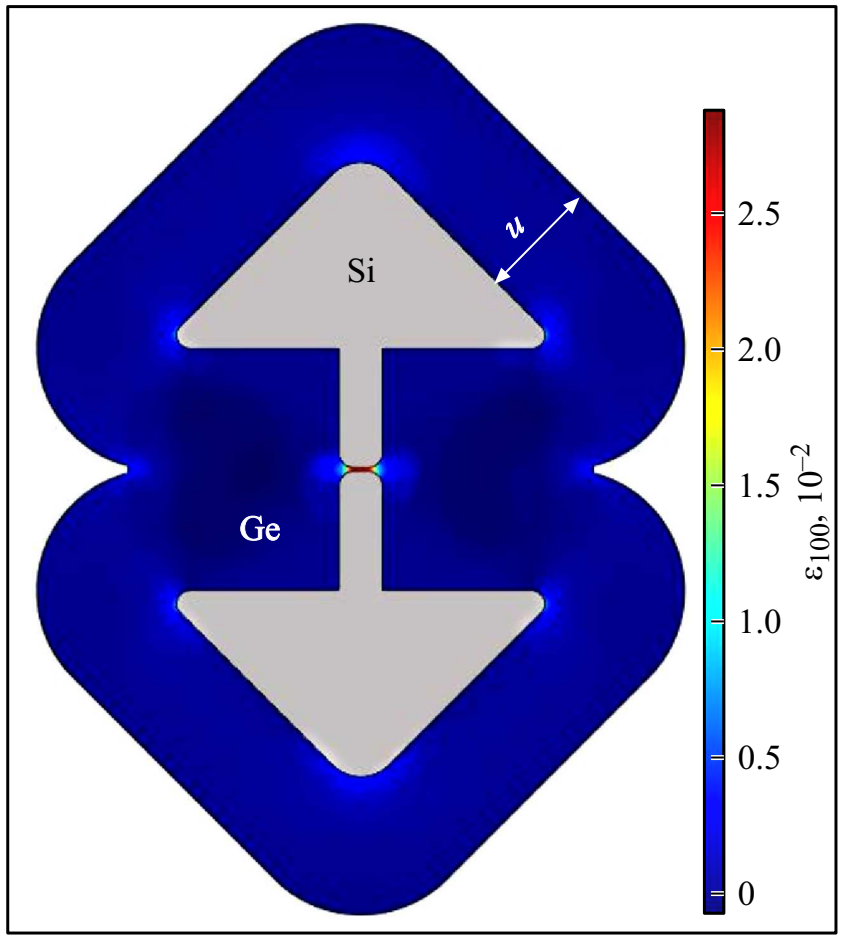

Рис. 2. Результаты расчета распределения деформации в Ge-микромостиках при их подвешивании за счет анизотропного травления $\mathrm{Si}(a)$ или изотропного травления $\mathrm{SiO}_{2}(b)$. Стрелками на рисунке $(a)$ обозначены кристаллографические направления. Буквами обозначены: $L$ - линейный размер (длина) формируемой ПХТ микроструктуры, $W$ - ширина „берега“, $u$ - ширина подвешенной части Ge-пленки вне микромостика. Представленное распределение деформации рассчитано для Ge-микромостиков толщиной $900 \mathrm{HM} \mathrm{c}$ исходной деформацией растяжения $\varepsilon_{0}=0.25 \%, L=250$ мкм, $W=150$ мкм, $u=50$ мкм, шириной микромостика $w=3$ мкм, длиной $l=10$ мкм и радиусом закругления в углах структуры $r=8$ мкм. Ширина подвешенной части Ge-пленки вне микромостика $u=0.5$ мкм для случая анизотропного травления $(a)$ и $u=W / 2=75$ мкм для изотропного травления $(b)$. 
увеличением $\varepsilon_{0}, L$ и $W$ и с уменьшением $w$ и $l$. Кроме этого, величина деформации мостика увеличивается с

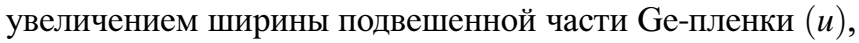
окружающей микромостик и „берега“ (рис. 2, b). В случае использования в качестве исходных структур с $\mathrm{Ge}$-слоями, выращенными на $\mathrm{Si}(001)$-подложках, дизайн микроструктур, показанный на рис. 1 и 2, позволяет минимизировать величину $u$ (рис. 1 и $2, a$ ) за счет того, что внешний размер всей микроструктуры ограничен плоскостями (111), для которых скорость травления кремния в КОН растворах является минимальной (см., например, [21]). Это дает возможность точного контроля максимальной деформации в мостике, которая в этом случае определяется только размерами микроструктуры, сформированной с помощью фотолитографии и ПХТ. При использовании в качестве исходных структур, выращенных на SOI подложках, изотропное травление слоя скрытого окисла позволяет увеличивать деформацию в мостике за счет бокового травления окисла, т.е. увеличения значения параметра $u$ (рис. 2,b). При этом минимальное значение $\mathrm{u}$ при полном подвешивании „берегов“ составляет $u=W / 2$ (рис. 2, $b$ ). Так, расчеты распределения деформации в микромостиках с параметрами $L=250$ мкм, $W=150$ мкм, $w=3$ мкм, $l=10$ мкм, представленные на рис. 2 , показывают, что при переходе от анизотропного травления к изотропному одноосное растяжение мостика может быть увеличено с $\sim 2.4 \%$ до $2.7 \%$ (рис. 2 ).

Теоретический анализ распределения напряжений в формируемых Ge-микроструктурах показал, что для достижения максимальной концентрации напряжений растяжения в микромостике необходимо минимизировать концентрацию деформаций во всех углах формируемой структуры. Существенное уменьшение концентрации деформации в углах происходит при увеличении радиуса их закругления $(r)$ до значений в несколько микрон (рис. 1 и 2). В противном случае перераспределение упругих напряжений при подвешивании микроструктуры приводит к разрыву пленки Ge в углах структуры с максимальной концентрацией напряжений (в острых углах с наименьшим радиусом закругления). Кроме того, увеличение $r$ в местах примыкания микромостика к „берегам“ позволяет значительно улучшить однородность распределения деформации в мостике.

Исследования полученных $\mathrm{Ge}$-микромостиков методом микро-КРС на зондовой нанолаборатории NTEGRA Spectra позволило получить как снимки самого микромостика, так и одномерные и двумерные карты спектрального положения пика LO-фонона в Ge. B качестве примера на рис. 3 показан снимок Ge-микромостика и совмещенный с ним скан положения максимума пика $\mathrm{LO}-ф о н о н а \mathrm{Ge}$ вдоль пунктирной линии. Из смещения $(\Delta \omega)$ линии LO-фонона Ge в структуре относительно этой линии в объемном, недеформированном Ge может быть вычислено значение локальной деформации в микроструктуре (рис. 3 и 4). В данной работе для вычисления одноосной деформации микромостиков вдоль

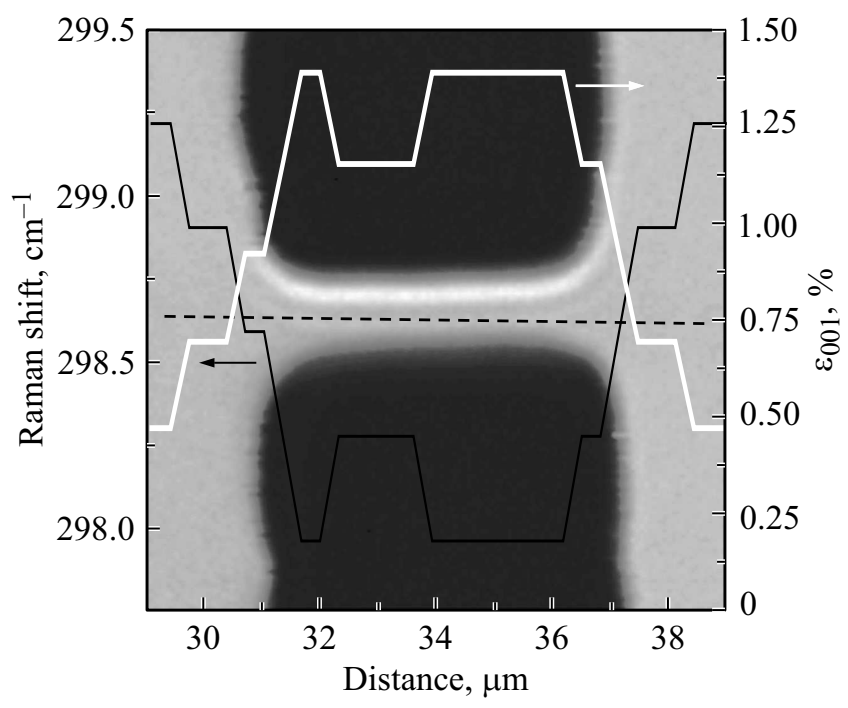

Рис. 3. Снимок Ge-микромостика, полученный с помощью зондовой микроскопии, и совмещенный с ним скан (вдоль пунктирной линии) положения линии LO-фонона $\mathrm{Ge}$ в микромостике (левая ось) и вычисленное из этого положения распределение деформации в нем (правая ось).

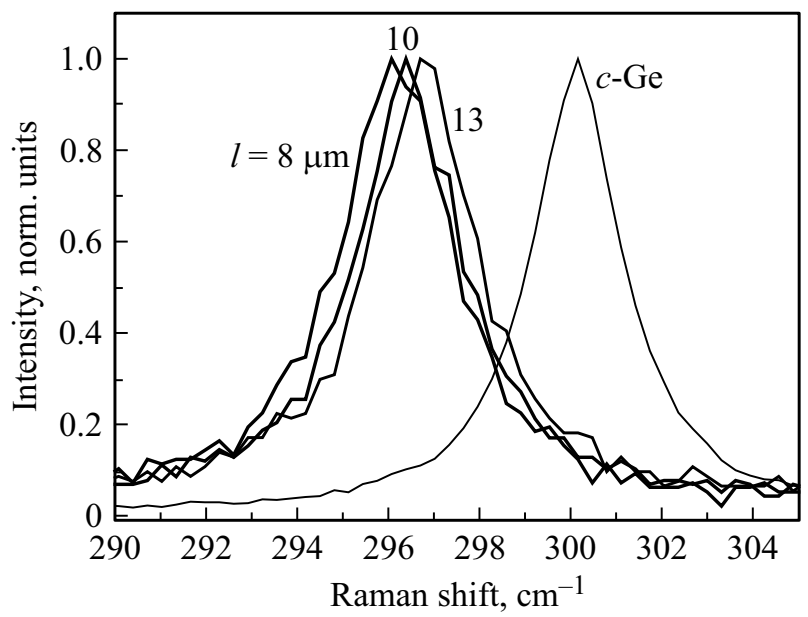

Pис. 4. Спектры микро-КРС в окрестности линии LO-фонона $\mathrm{Ge}$, измеренные на объемном, недеформированном $\mathrm{Ge}(c-\mathrm{Ge})$ и в центре микромостиков длиной $l=8,10$ и 13 мкм. Сформированные мостики имели параметры: $L=250$ мкм, $W=150$ мкм, $w \sim 3$ мкм, $r=8$ мкм и $u \sim 0$.

направления [100] использовалась формула

$$
\varepsilon=0.68 \Delta \omega-0.019 \Delta \omega^{2},
$$

полученная в работе [17] и учитывающая отклонение от линейной связи $\varepsilon$ и $\Delta \omega$. Из рис. 3 и 4 видно, что в результате подвешивания происходит многократное возрастание одноосной деформации в микромостике по сравнению с исходной, однородной, двумерной деформацией в $\mathrm{Ge} / \mathrm{Si}(001)$-пленке. При этом деформация уменьшается с увеличением длины мостиков (рис. 4). Полученные из спектров микро-КРС значения дефор- 
мации растяжения микромостиков хорошо согласуются с результатами теоретических расчетов распределения деформации в них. Максимально достигнутое в данной работе одноосное растяжение составило $\sim 2.4 \%$, что на порядок превосходит исходную деформацию в $\mathrm{Ge} / \mathrm{Si}$ пленке. При большей величине растяжения происходит разрыв микромостиков, который может быть вызван наличием в подвешиваемой части структуры дефектной области, располагающейся на границе $\mathrm{Si}$ и Ge.

Исследования излучательных свойств полученных Ge-микроструктур при комнатной температуре методом спектроскопии микро-ФЛ в режиме непрерывной накачки (длина волны возбуждения 532 нм, пространственное разрешение $\sim 2$ мкм) показали значительное возрастание интенсивности сигнала ФЛ от Ge-мостика, т.е. от области с максимальной деформацией растяжения, по сравнению с исходным, слабо растянутым Ge-слоем (рис. 5). В спектре микро-ФЛ исходной, нелегированной $\mathrm{Ge} / \mathrm{SOI}$-структуры присутствует как пик, связанный с рекомбинацией электронов из Г-долины (прямые переходы, пик в районе 1560 нм), так и пик, вызванный рекомбинацией электронов из $L$-долин (непрямые переходы в области 1790 нм) (спектр 1 на рис. 5). После формирования микроструктуры наблюдается рост сигнала ФЛ как от области подвешенной Ge-пленки вблизи микромостика („берега“) (спектр 2 на рис. 5), так и от самого микромостика (спектр 3 на рис. 5). При этом вид спектра ФЛ от „берега“ в отличие от спектра ФЛ микромостика существенно не отличается от спектра исходной Ge-пленки. Можно отметить небольшое смещение пика ФЛ, связанного с прямыми переходами в „береге ““, в сторону меньших длин волн по сравнению с исходной Ge-пленкой, которое может быть вызвано релаксацией упругих напряжений растяжения в подвешенной части пленки. Рост интенсивности сигнала ФЛ от „берега“ по сравнению с „непроцессированной“ областью связывается с более эффективным выводом

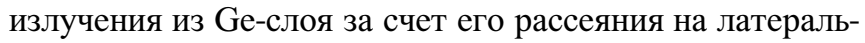
ных границах микроструктуры.

Спектр ФЛ Ge-микромостика существенно отличается как от спектра ФЛ исходной Ge-пленки, так и от спектра ФЛ „берегов“" (рис. 5). Можно отметить значительное возрастание интегральной интенсивности ФЛ при приближении к мостику (см. вставку на рис. 5) и смещение его максимума в более длинноволновую область. Оба этих изменения в спектре ФЛ связываются с ростом деформации растяжения в микромостике, который приводит как к уменьшению ширин прямой и непрямой запрещенных зон $\mathrm{Ge}$, так и к уменьшению энергетического зазора между ними. Именно уменьшение энергетического расстояния между Г- и $L$-долинами, по-видимому, вызывает рост интенсивности ФЛ микромостика за счет увеличения вклада в сигнал ФЛ прямых излучательных переходов из Г-долины.

Следует отметить, что наряду с описанным выше механизмом рост интенсивности ФЛ Ge-микромостиков

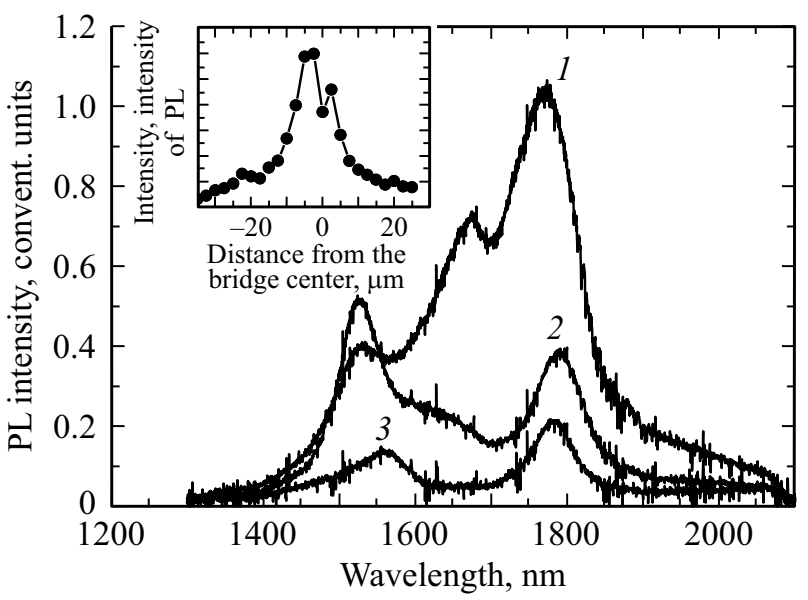

Рис. 5. Спектры микро-ФЛ структуры с Ge-микромостиком при непрерывной накачке: $1-$ в области максимальной деформации растяжения вблизи центра мостика, 2 - на подвешенной области Ge-слоя вблизи мостика („берег“), 3 - на непроцессированном участке Ge-слоя вдали от мостика. На вставке - интегральная интенсивность ФЛ в зависимости от удаления пятна возбуждения от центра мостика.

может быть вызван локальным разогревом свободновисящих мостиков при их возбуждении интенсивным непрерывным лазерным излучением. Для выявления возможного вклада в увеличение сигнала ФЛ эффектов разогрева были проведены исследования спектров микро-ФЛ структур с Ge-мостиками в условиях импульсного оптического возбуждения фемтосекундным лазером (длина волны - 800 нм, длительность импульса $\sim 200$ фс, частота повторения импульсов -50 кГц). Короткая длительность и относительно низкая частота повторения импульсов накачки позволяли избежать заметного разогрева мостиков. Спектры ФЛ, полученные в режиме импульсной накачки, также продемонстрировали заметное возрастание интегральной интенсивности сигнала ФЛ в области Ge-мостиков. Данный факт позволяет связать наблюдаемый рост интенсивности сигнала ФЛ в полученных локально-растянутых Ge-микроструктурах с ростом вероятности прямых оптических переходах в Ge при его растяжении.

\section{4. Заключение}

В работе представлены результаты по формированию и исследованию локально-растянутых Ge-микроструктур на основе слоев $\mathrm{Ge}$, выращенных на подложках $\mathrm{Si}(001)$ и „кремний-на-изоляторе“. Выполнен теоретический анализ распределения деформации в свободновисящих Ge-микромостиках, который выявил необходимость минимизации концентрации упругих напряжений в углах формируемых структур для достижения максимального уровня деформации растяжения в самом микромостике. Измерения локальной деформации методом комбинационного рассеяния света показали увеличение дефор- 
мации растяжения с $0.2-0.25 \%$ в исходной Ge-пленке до $\sim 2.4 \%$ в микромостиках. Методом спектроскопии микрофотолюминесценции продемонстрировано значительное возрастание интенсивности и существенная модификация спектра ФЛ в области максимальных растягивающих напряжений в Ge-микромостиках и их окрестности по сравнению со слабо растянутыми участками исходных слоев $\mathrm{Ge}$.

Работа частично выполнена в рамках государственного задания ИФМ РАН (тема 0035-2014-0201), при финансовой поддержке РФФИ (проект № 16-29-14056офи_м). Работа выполнена с использованием оборудовании УСУ „Фемтоспектр“ ЦКП ИФМ РАН.

\section{Список литературы}

[1] S. Saito, A.Z. Al-Attili, K. Oda, Y. Ishikawa. Semicond. Sci. Technol., 31, 043002 (2016).

[2] R. Geiger, T. Zabel, H. Sigg. Frontiers Mater., 2, 52 (2015).

[3] J. Liu, X. Sun, D. Pan, X. Wang, L.C. Kimerling, T.L. Koch, J. Michel. Opt. Express, 15, 11272 (2007).

[4] J. Menéndez, J. Kouvetakis. Appl. Phys. Lett., 85, 1175 (2004).

[5] M. Virgilio, C.L. Manganelli, G. Grosso, G. Pizzi, G. Capellini. Phys. Rev. B, 87, 235313 (2013).

[6] Y.-Y. Fang, J. Tolle, R. Roucka, A.V.G. Chizmeshya, J. Kouvetakis. Appl. Phys. Lett., 90, 061915 (2007).

[7] G. Capellini, M. De Seta, P. Zaumseil, G. Kozlowski, T. Schroeder. J. Appl. Phys., 111, 73518 (2012).

[8] R.A. Minamisawa, M.J. Süess, R. Spolenak, J. Faist, C. David, J. Gobrecht, K.K. Bourdelle, H. Sigg. Nature Commun., 3, 1096 (2012).

[9] M.J. Süess, R. Geiger, R.A. Minamisawa, G. Schiefler, J. Frigerio, D. Chrastina, G. Isella, R. Spolenak, J. Faist, H. Sigg. Nature Photonics, 7, 466 (2013).

[10] S. Bao, D. Kim, C. Onwukaeme, S. Gupta, K. Saraswat, K.H. Lee, Y. Kim, D. Min, Y. Jung, H. Qiu, H. Wang, E.A. Fitzgerald, C.S. Tan, D. Nam. Nature Commun., 8, 1845 (2017).

[11] H.-C. Luan, D.R. Lim, K.K. Lee, K.M. Chen, J.G. Sandland, K. Wada, L.C. Kimerling. Appl. Phys. Lett., 75, 2909 (1999).

[12] L. Colace, G. Masini, F. Galluzzi, G. Assanto, G. Capellini, L. Di Gaspare, E. Palange, F. Evangelisti. Appl. Phys. Lett., 72, 3175 (1998).

[13] J.-M. Hartmann, A. Abbadie, J.P. Barnes, J.M. Fedeli, T. Billon, L. Vivien. J. Cryst. Growth, 312, 532 (2010).

[14] Д.В. Юрасов, А.И. Бобров, В.М. Данильцев, А.В. Новиков, Д.А. Павлов, Е.В. Скороходов, М.В. Шалеев, П.А. Юнин. ФТП, 49, 1463 (2015).

[15] D.V. Yurasov, A.V. Antonov, M.N. Drozdov, V.B. Schmagin, K.E. Spirin, A.V. Novikov. J. Appl. Phys., 118, 145701 (2015).

[16] Н.А. Байдакова, В.А. Вербус, Е.Е. Морозова, А.В. Новиков, Е.В. Скороходов, М.В. Шалеев, Д.В. Юрасов, А. Ноmbe, Y. Kurokawa, N. Usami. ФТП, 51 (12), 1599 (2017).

[17] A. Gassenq, S. Tardif, K. Guilloy, I. Duchemin, N. Pauc, J.-M. Hartmann, D. Rouchon, J. Widiez, Y.M. Niquet, L. Milord, T. Zabel, H. Sigg, J. Faist, A. Chelnokov, F. Rieutord, V. Reboud, V. Calvo. J. Appl. Phys., 121, 055702 (2017).
[18] J.J. Wortman. R.A. Evans. J. Appl. Phys., 36, 153 (1965).

[19] Guo-En Chang, H.H. Cheng. J. Phys. D: Appl. Phys., 46, 065103 (2013).

[20] G. Capellini, C. Reich, S. Guha, Y. Yamamoto, M. Lisker, M. Virgilio, A. Ghrib, M. El Kurdi, P. Boucaud, B. Tillack, T. Schroeder. Opt. Express, 22, 399 (2014).

[21] K.P. Rola, I. Zubel. Mater. Sci. Poland, 29, 278 (2011).

Редактор Г.А. Оганесян

\section{Formation and properties of locally strained Ge microstructures for silicon photonics}

\author{
A.V. Novikov ${ }^{\mathbf{1}, 2}$, D.V. Yurasov ${ }^{\mathbf{1}, 2}$, E.E. Morozova ${ }^{1}$, \\ E.V. Skorohodov ${ }^{1}$, V.A. Verbus ${ }^{1,3}$, A.N. Yablonskiy ${ }^{1}$, \\ N.A. Baidakova ${ }^{1}$, N.S. Gusev' ${ }^{1}$, K.E. Kudryavtsev ${ }^{1,2}$, \\ A.V. Nezhdanov ${ }^{2}$, A.I. Mashin ${ }^{2}$ \\ ${ }^{1}$ Institute for Physics of Microstructures, \\ Russian Academy of Sciences, \\ 603087 Nizhny Novgorod region, Russia \\ ${ }^{2}$ Lobachevsky State University of Nizhny Novgorod, \\ 603950 Nizhny Novgorod, Russia \\ ${ }^{3}$ National Research University \\ Higher School of Economics, \\ 603155 Nizhny Novgorod, Russia
}

\begin{abstract}
The results of the formation and study of locally tensile-strained $\mathrm{Ge}$ microstructures based on Ge layers grown on silicon substrates are presented. A theoretical analysis of the deformation distribution in free-standing Ge microbridges was carried out, which revealed the need to minimize the concentration of elastic stresses in the corners of the formed microstructures in order to achieve the maximum tensile strain in the microbridges. Measurements of local deformation using Raman scattering showed significant increase of the tensile strain from $0.2-0.25 \%$ in the initial Ge film up to $\sim 2.4 \%$ in Ge microbridges. The micro-photoluminescence spectroscopy demonstrated remarkable increase of the luminescence intensity and modification of the spectrum in the regions of the maximum tensile strain in $\mathrm{Ge}$ microbridges and their vicinity as compared to the slightly strained regions of the initial Ge film.
\end{abstract}

